\title{
Nature of superfluidity in ultracold Fermi gases near Feshbach resonances
}

\author{
Jelena Stajic, ${ }^{1}$ J. N. Milstein, ${ }^{2}$ Qijin Chen, ${ }^{3}$ M. L. Chiofalo, ${ }^{4}$ M. J. Holland, ${ }^{2}$ and K. Levin ${ }^{1}$ \\ ${ }^{1}$ James Franck Institute and Department of Physics, University of Chicago, Chicago, Illinois 60637, USA \\ ${ }^{2}$ JILA, University of Colorado and National Institute of Standards and Technology, Boulder, Colorado 80309, USA \\ ${ }^{3}$ Department of Physics and Astronomy, Johns Hopkins University, Baltimore, Maryland 21218, USA \\ ${ }^{4}$ Classe di Scienze and INFM, Scuola Normale Superiore, Piazza dei Cavelieri 7, I-56126 Pisa, Italy
}

(Received 18 February 2004; published 17 June 2004)

\begin{abstract}
We study the superfluid state of atomic Fermi gases using a BCS-Bose-Einstein-condensation crossover theory. Our approach emphasizes noncondensed fermion pairs which strongly hybridize with their (Feshbachinduced) molecular boson counterparts. These pairs lead to pseudogap effects above $T_{c}$ and non-BCS characteristics below. We discuss how these effects influence the experimental signatures of superfluidity.
\end{abstract}

DOI: 10.1103/PhysRevA.69.063610

There has been considerable interest in achieving "resonance superfluidity" in an ultracold, extremely dilute, trapped Fermi gas in which a Feshbach resonance [1,2] is used to tune the interatomic attraction by variation of a magnetic field. These systems allow for the direct study of the crossover problem where the nature of the superfluid transition changes from BCS to Bose-Einstein condensation (BEC). Recently, several groups have observed superfluidity in the BEC regime [3-5]. This provides an exciting opportunity for theoretical work which can address the entire range of behavior, in anticipation of future experiments.

In this paper we consider the case of a homogeneous gas as a first and necessary step. Our goals are to present a theory for $T_{c}$, for the superfluid phase as well as some signatures of the transition, which are shown to be very different from conventional expectations based on BCS theory. Using current understanding of bosonic superfluidity, it is useful to begin with a number of inferences about the nature of the superfluidity induced when molecular bosons are admixed with fermions. For temperatures $T$ above $T_{c}$ sufficiently strong hybridization $\left(g b_{\mathbf{q}}^{\dagger} a_{\mathbf{q}-\mathbf{k}} a_{\mathbf{k}}\right)$ between molecular bosons $\left(b_{\mathbf{q}}^{\dagger}\right)$ and fermion pairs will result in metastable "preformed" pairs $\left(a_{\mathbf{q}-\mathbf{k}}^{\dagger} a_{\mathbf{k}}^{\dagger}\right)$. These are necessarily associated with a normal state excitation gap which represents the energy needed to break them apart into fermionic quasi-particles $\left(a_{\mathbf{k}}^{\dagger}\right)$. For $0<T \leqslant T_{c}$ the presence of noncondensed molecular bosons similarly induces the formation of noncondensed fermion pairs. These must be present in addition to the usual fermionic single particle excitations of the condensate. We may, thus, quite generally infer that since the molecular bosons and the fermion pairs are so strongly entangled, they must be treated on a similar footing.

Previous [6,7] studies of the crossover problem in an atomic Fermi gas at $T \neq 0$ have been based on the work [8] of Nozières and Schmitt-Rink (NSR). This approach, which effectively omits noncondensed fermion pairs, addresses $T_{c}$ in a fashion which is not manifestly compatible with the presumed ground state [9]. Moreover, pairing correlations are only included in the number equation and within an approximate and evidently $[10,11]$ problematic fashion. In this paper we address these shortcomings, but similarly base our analysis of the standard BCS-like crossover ground state [9], for the fermionic component. We begin with the important
PACS number(s): 03.75.-b, 64.60.-i, 74.20.-z

observation that the noncondensed fermion pairs are in chemical equilibrium with both the noncondensed molecular bosons and the condensate. Consequently, they must satisfy

$$
\mu_{\text {pair }}(T)=\mu_{\text {boson }}(T)=0, \quad T \leqslant T_{c} .
$$

This last relation, which is a central equation of this paper, will be rewritten shortly in a more concrete form. To implement the physical picture discussed above, we follow the theory in Ref. [12], extending it to include a Feshbach resonance. This approach was originally developed to treat high $T_{c}$ superconductors. The physics in the context of bosonfermion models is shown here to be different from fermiononly models, although such hybridization models have been also applied to the cuprates $[13,14]$.

Our Hamiltonian [1] is given by

$$
\begin{aligned}
H-\mu N= & \sum_{\mathbf{k}, \sigma} \epsilon_{\mathbf{k}} a_{\mathbf{k}, \sigma}^{\dagger} a_{\mathbf{k}, \sigma}+\sum_{\mathbf{q}}\left(\epsilon_{\mathbf{q}}^{m}+\nu\right) b_{\mathbf{q}}^{\dagger} b_{\mathbf{q}} \\
& +\sum_{\mathbf{q}, \mathbf{k}, \mathbf{k}^{\prime}} U\left(\mathbf{k}, \mathbf{k}^{\prime}\right) a_{\mathbf{q} / 2+\mathbf{k}, \uparrow}^{\dagger} a_{\mathbf{q} / 2-\mathbf{k}, \downarrow}^{\dagger} a_{\mathbf{q} / 2-\mathbf{k}^{\prime}, \downarrow} a_{\mathbf{q} / 2+\mathbf{k}^{\prime}, \uparrow} \\
& +\sum_{\mathbf{q}, \mathbf{k}}\left(g(\mathbf{k}) b_{\mathbf{q}}^{\dagger} a_{\mathbf{q} / 2-\mathbf{k}, \downarrow} a_{\mathbf{q} / 2+\mathbf{k}, \uparrow}+\text { H.c. }\right) .
\end{aligned}
$$

The sum in $\sigma$ runs over both spin states $\{\uparrow, \downarrow\}$. The free dispersion relations for fermions and bosons are given by and $\epsilon_{\mathbf{q}}^{m}=E_{\mathbf{q}}^{0}-2 \mu$ with $E_{\mathbf{q}}^{0}=q^{2} / 2 M$, respectively, where we assume $\mu_{\uparrow}=\mu_{\downarrow}$, and $M=2 m$ is the boson mass. Here $\nu$ is the detuning of the resonance state, $U\left(\mathbf{k}, \mathbf{k}^{\prime}\right)=U \varphi_{\mathbf{k}} \varphi_{\mathbf{k}^{\prime}}$ is the direct $s$-wave interaction and $g(\mathbf{k})=g \varphi_{\mathbf{k}}$ is the Feshbach coupling, with the function $\varphi_{\mathbf{k}}^{2}=\exp \left\{-\left(k / K_{c}\right)^{2}\right\}$ providing the momentum cutoff. Here we set $\hbar=k_{B}=1$.

The three propagators for fermion pairs, $t(Q)$, molecular bosons, $D(Q)$, and single fermions, $G(Q)$ are coupled. (Throughout we take the convention $\Sigma_{K} \equiv T \Sigma_{\omega_{n}} \Sigma_{\mathbf{k}}$, where $K, Q$, etc. are 4-vectors.) With these definitions Eq. (1) can also be rewritten

$$
D^{-1}(0)=t^{-1}(0)=0, \quad T \leqslant T_{c} .
$$

The effective pairing interaction is given by [7] $\tilde{g}_{\text {eff }}\left(Q, K, K^{\prime}\right)=g_{\text {eff }}(Q) \varphi_{\mathbf{k}} \varphi_{\mathbf{k}^{\prime}}$ with $g_{\text {eff }}(Q) \equiv U+g^{2} D_{0}(Q)$, where $D_{0}(Q) \equiv 1 /\left[i \Omega_{n}-E_{q}^{0}-\nu+2 \mu\right]$ is the noninteracting 
molecular boson propagator with Matsubara frequency $\Omega_{n}$. We have from Eq. (3) and the general form of the $T$ matrix the important result that

$$
t^{-1}(0) \equiv g_{\text {eff }}^{-1}(0)+\chi(0)=0, \quad T \leqslant T_{c} .
$$

We now impose the reasonable constraints that at weak coupling our results are compatible with the $T$ dependence found in the BCS limit for $\Delta(T)$, and for general coupling, the fermions are described by the standard $T=0$ crossover state [9]. This, in turn, constrains the fermion pair susceptibility $\chi(Q)$,

$$
\chi(Q)=\sum_{K} G(K) G_{0}(Q-K) \varphi_{\mathbf{k}-\mathbf{q} / 2}^{2},
$$

provided also the fermion self-energy appearing in $G$ is

$$
\Sigma(K)=\sum_{Q} t(Q) G_{0}(Q-K) \varphi_{\mathbf{k}-\mathbf{q} / 2}^{2} .
$$

This form for $\chi$ and $\Sigma$ can also be derived by truncating the equations of motion for Green's functions at the threeparticle level. This approach is also closely related to Hartree-approximated time dependent Ginzburg-Landau theory [15].

The $T$ matrix consists of two contributions: from the condensed or superconducting $(s c)$ and noncondensed or "pseudogap"-associated ( $\mathrm{pg}$ ) pairs. The molecular bosons also contribute to the $T$ matrix via the effective pairing interaction:

$$
\begin{gathered}
t=t_{p g}+t_{s c}, \\
t_{p g}(Q)=\frac{g_{e f f}(Q)}{1+g_{\text {eff }}(Q) \chi(Q)}, \quad Q \neq 0, \\
t_{s c}(Q)=-\frac{\widetilde{\Delta}_{s c}^{2}}{T} \delta(Q),
\end{gathered}
$$

where $\widetilde{\Delta}_{s c}=\Delta_{s c}-g \phi_{m}$, with $\Delta_{s c}=-U \Sigma_{\mathbf{k}}\left\langle a_{-\mathbf{k} \downarrow} a_{\mathbf{k} \uparrow}\right\rangle \varphi_{\mathbf{k}}$ and $\phi_{m}$ $=\left\langle b_{\mathbf{q}=0}\right\rangle$. Without loss of generality, we choose order parameters $\widetilde{\Delta}_{s c}$ and $\phi_{m}$ to be real and positive with $g<0$. The order parameter is a linear combination of both paired atoms and condensed molecules. These two components are connected [16] by the relation $\phi_{m}=g \Delta_{s c} /[(\nu-2 \mu) U]$. This implies that $\widetilde{\Delta}_{s c}=-g_{\text {eff }}(0) \Sigma_{\mathbf{k}}\left\langle a_{-\mathbf{k} \downarrow} a_{\mathbf{k} \uparrow}\right\rangle \varphi_{\mathbf{k}}$, as expected. Following Eq. (3), for $T \leqslant T_{c}$ we may approximate [17] Eq. (6) to yield a BCSlike self-energy with $\Delta^{2} \equiv \widetilde{\Delta}_{s c}^{2}+\Delta_{p g}^{2}$,

$$
\Sigma(K) \approx-G_{0}(-K) \Delta^{2} \varphi_{\mathbf{k}}^{2},
$$

where we define the pseudogap $\Delta_{p g}$

$$
\Delta_{p g}^{2} \equiv-\sum_{Q \neq 0} t_{p g}(Q) .
$$

At $T=0, \Delta_{p g}=0$, so that $\widetilde{\Delta}_{s c}(0)=\Delta(0)$. Equation (4) can be written as

$$
g_{\text {eff }}^{-1}(0)+\sum_{\mathbf{k}} \frac{1-2 f\left(E_{\mathbf{k}}\right)}{2 E_{\mathbf{k}}} \varphi_{\mathbf{k}}^{2}=0, \quad T \leqslant T_{c},
$$

where $E_{\mathbf{k}}=\sqrt{\epsilon_{\mathbf{k}}^{2}+\Delta^{2} \varphi_{\mathbf{k}}^{2}}$. Equation (12) has the form of the conventional BCS equation, but the full excitation gap $\Delta$, as distinguished from the order parameter, $\widetilde{\Delta}_{s c}$, appears in the dispersion $E_{\mathbf{k}}$.

We show next that this equation for the excitation gap $\Delta(T)$ coincides with the equivalent condition on the molecular bosons, given in Eq. (3). Taking the same pair susceptibility $\chi$ in the boson self-energy $\Sigma_{B}$, we obtain

$$
\Sigma_{B}(Q) \equiv-g^{2} \chi(Q) /[1+U \chi(Q)]
$$

so that the boson propagator is

$$
D(Q) \equiv \frac{1}{i \Omega_{n}-E_{q}^{0}-\nu+2 \mu-\Sigma_{B}(Q)} .
$$

After some algebra, Eq. (3) leads directly to Eq. (12). We may now calculate the number equation from the propagators involved. The number of noncondensed molecular bosons is given directly by $n_{b}=-\Sigma_{Q} D(Q)$. For $T \leqslant T_{c}$, the number of fermions is

$$
n_{f}=\sum_{\mathbf{k}}\left[1-\frac{\epsilon_{\mathbf{k}}}{E_{\mathbf{k}}}+2 \frac{\epsilon_{\mathbf{k}}}{E_{\mathbf{k}}} f\left(E_{\mathbf{k}}\right)\right],
$$

as follows from the condition $n_{f}=2 \Sigma_{K} G(K)$ with a BCS-like self-energy $\Sigma(K)$. The total number $(n)$ of fermions is then

$$
n_{f}+2 n_{b}+2 n_{b}^{0}=n,
$$

where $n_{b}^{0}=\phi_{m}^{2}$ is the number of molecular bosons in the condensate.

We now have a closed set of equations for our resonance system which requires a numerical solution. The cutoff function $\varphi_{\mathbf{k}}$ introduces a renormalization of $U, g$, and $\nu$. Extending the derivation given in Ref. [16] to a nonconstant, separable potential, we find that $U=\Gamma U_{0}, g=\Gamma g_{0}$, and $\nu=\nu_{0}$ $+\alpha \Gamma g_{0}^{2}$. Here $U_{0}=4 \pi a_{b g} / m<0$ where $a_{b g}$ is the background scattering length and $g_{0}$ is the physical scattering parameter reflecting the width of the Feshbach resonance. The scaling factor $\Gamma=1 /\left(1-\alpha U_{0}\right)$ and $\alpha=m K_{c} /\left(4 \pi^{3 / 2}\right)$.

At the physical level, the essential distinction between this and previous crossover studies based on the NSR approach [18] below $T_{c}$ is associated with noncondensed pair excitations [19] of the superfluid. At a more formal level, it should be noted that there is an important difference between Eq. (5) and previous related work [8]. This NSR approach presumes that there are two bare Green's functions $G_{0}$ in $\chi(Q)$ : the particles acquire a self-energy from the pairs, but these self-energy effects are not fed back into the propagator for the pairs.

To evaluate $T_{c}, \Delta(T), \mu(T)$, as well as $\widetilde{\Delta}_{s c}(T)$ and other transport properties $[12,15]$ below $T_{c}$, we solve Eqs. (11), (12), and (16) for fixed $U_{0}, g_{0}$, and $\nu_{0}$ and a sufficiently large cutoff $K_{c}$. Due to the divergence of the $T$ matrix [Eq. (4)], we may Taylor expand the quantity $\chi(Q)$ in Eqs. (16) and (11) to 


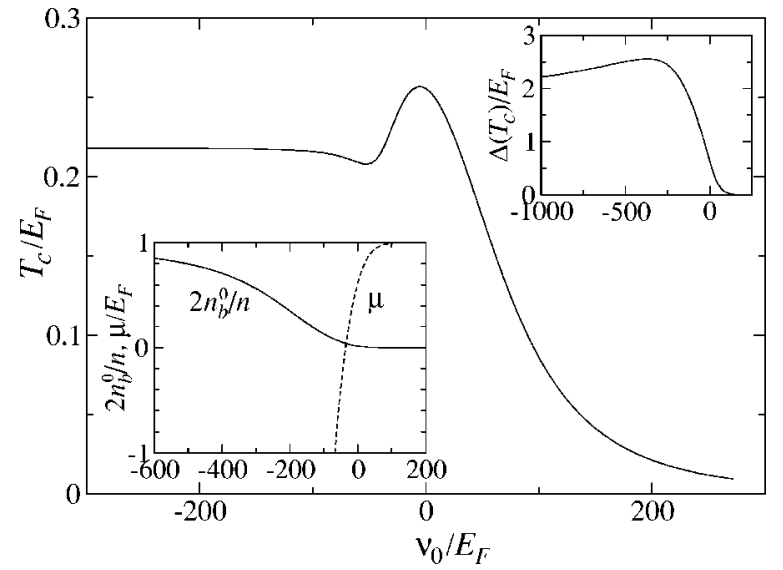

FIG. 1. $T_{c}$ vs detuning $\nu_{0}$. The upper inset plots the pseudogap at $T_{c}$ vs $\nu_{0}$, and the lower inset plots molecular boson contribution to the condensate weight and fermionic chemical potential at $T=0$.

first order in $\Omega$ and $q^{2}: \chi(Q)=\chi(0)+a_{0}\left(i \Omega_{n}-B_{0} q^{2}\right)$ [15], and expand $g_{\text {eff }}(Q)$ and $\Sigma_{B}(Q)$ similarly. This considerably simplifies the analysis.

We begin with calculations of $T_{c}$ as a function of $\nu_{0}$ which are plotted in Fig. 1. Here we take $g_{0}=-42 E_{F} / k_{F}^{3 / 2}$ and $U_{0}$ $=-3 E_{F} / k_{F}^{3}$ as is expected to be reasonably indicative of the behavior for $T_{c}$ in currently trapped atomic Fermi gases. For $\nu_{0} \rightarrow-\infty$ only molecular bosons are present and $T_{c}$ approaches the ideal BEC limit. As $\nu_{0} \rightarrow+\infty$, molecular bosons become irrelevant and the asymptote of the curve is dictated by the behavior of fermions in the presence of $U$. In this paper we have chosen $U_{0}$ deliberately to be small so that the $\nu_{0} \rightarrow \infty$ limit is close to BCS.

Indicated in the upper right inset is the behavior of $\Delta\left(T_{c}\right)$ as a function of $\nu_{0}$. The lower left inset shows a plot of the molecular Bose condensate weight $n_{b}^{0}$, and the fermionic chemical potential $\mu$ as a function of $\nu_{0}$. For positive, but decreasing $\nu_{0}, T_{c}$ follows the BCS curve until the "pseudogap" or $\Delta\left(T_{c}\right)$ becomes appreciable. After its maximum (at slightly negative $\nu_{0}$ ), $T_{c}$ decreases, as does $\mu$, until $\mu=0$. Beyond this point, towards negative $\nu_{0}$, the system is effectively bosonic. The condensate consists of two contributions, although the weight of the fermion pair component rapidly disappears, as can be inferred from the lower inset. Similarly $T_{c}$ rises, although slowly, towards the ideal BEC asymptote, following the inverse effective boson mass. The corresponding curve based on the NSR approach has only one extremum, but nevertheless the overall magnitudes are not so different [6,7]. There are, however, key differences between the behavior of the fermionic excitation gap $\Delta\left(T_{c}\right)$ and its high $T_{c}$ counterpart. Because all of the condensate comes from molecular bosons in the strict BEC limit, $\Delta\left(T_{c}\right)$ reaches a maximum (where the molecular bosonic and fermionic weights are comparable), and then decreases, as $\nu_{0}$ decreases. This is in contrast to fermion-only based models [12] where this parameter increases indefinitely as the attractive coupling becomes stronger.

In the inset to Fig. 2, we plot the temperature dependence of the normalized excitation gap $\Delta(T) / \Delta(0)$ for three values of $\nu_{0} / E_{F}=-200,-5,+200$. The second value corresponds

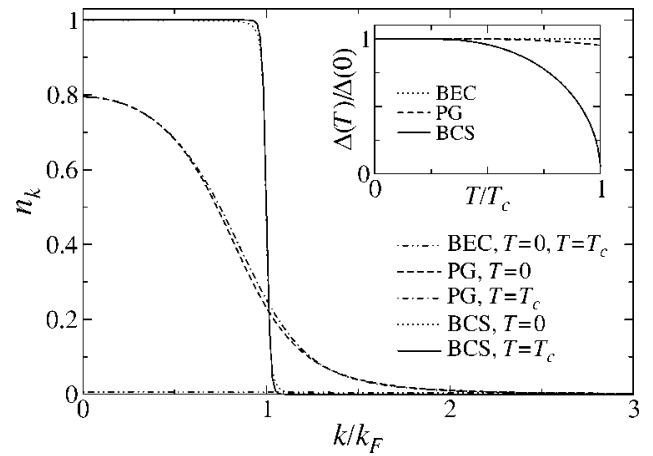

FIG. 2. Fermionic momentum distribution function at $T=0$ and $T=T_{c}$ for the three regimes. PG corresponds to maximal $T_{c}$. The inset plots the $T$ dependent excitation gap below $T_{c}$.

roughly to the maximum in $T_{c}$, where pseudogap effects are apparent. The first and last are illustrative of the BEC and BCS limits, respectively. We, thus, refer below to these three values as BEC, PG, and BCS cases. While not shown here, $\mu$ is positive for the latter two and negative for the first. The order parameter, $\widetilde{\Delta}_{s c}$, is not plotted here, but for all three cases it is rather close to the solid line in the inset. That $\Delta(T)$ is relatively constant with $T$ through the superfluid transition is to be expected in the presence of "preformed pairs," as for $\nu_{0} / E_{F}=-5$ and -200 .

It should be stressed that $T_{c}$ is only apparent in $\Delta(T)$ for the BCS case. To underline this point, in the main body of Fig. 2 we plot the fermionic momentum distribution function $n_{k}$ [20], which is the summand in Eq. (15), at $T=0$ and $T$ $=T_{c}$. The fact that there is very little change from $T=0$ to $T=T_{c}$ makes the important point that this momentum distribution function is not a good indicator of phase coherent pairing. For the PG case, this, in turn, derives from the fact that $\Delta(T)$ is nearly constant. For the BEC limit the excitation gap, which is dominated by $\mu$, similarly, does not vary through $T_{c}$. In the BCS regime, $\Delta(T)$ is sufficiently small as to be barely perceptible on the scale of the figure. In order to address the measurable particle density distribution, these observations will have to be incorporated into previous BCSbased (i.e., $\Delta \equiv \widetilde{\Delta}_{s c}$ ) calculations [21,22], albeit generalized to include inhomogeneity effects.

This pseudogap-based phenomenology is well documented in the high $T_{c}$ superconductors, although for these materials, penetration depth data (with no analog here) are direct probes of the transition to superconductivity. Interestingly, densities of state measurements in the cuprates also show some indications of when order is well established. To see how phase coherence enters in the atomic physics context, we relax the approximation in Eq. (10) by noting that incoherent or finite momentum pairs $(\mathrm{pg})$ are distinguishable from coherent or zero momentum pairs $(s c)$ through lifetime effects in the self-energy. For the $p g$ term we write $\Sigma_{p g}(\omega, \mathbf{k}) \approx \Delta_{p g}^{2} /\left(\omega+\epsilon_{\mathbf{k}}+i \gamma\right)$. For simplicity, we treat $\gamma$ as a phenomenological parameter which is independent of $T$. This distinction between $p g$ and $s c$ is required to arrive at general thermodynamic signatures (not discussed here) of the transition.

Figures 3(a)-3(c) show the resulting density of states $N(\omega)=-2 \Sigma_{\mathbf{k}} \operatorname{Im} G(\omega+i 0, \mathbf{k})$ and correspond to the BEC, 

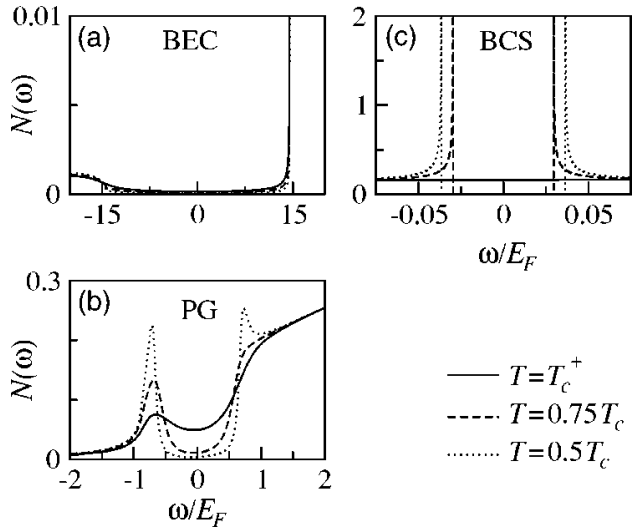

$$
\begin{aligned}
T & =T_{c}^{+} \\
-\ldots . T & =0.75 T_{c} \\
\ldots \ldots . T & =0.5 T_{c}
\end{aligned}
$$

FIG. 3. Fermionic density of states vs energy for the three regimes at three indicated temperatures. Note the difference in the scales.

PG, and BCS cases, as in Fig. 2. The temperatures shown are just above $T_{c}$, and for $T=0.75 T_{c}$ and $0.5 T_{c}$. The BCS case indicates an abrupt transition at $T_{c}$, but with a very small gap and very low $T_{c}$, which may be hard to observe experimentally. The BEC case shows very little temperature dependence throughout, since the fermions are well gapped at all temperatures. Only the PG case, where $T_{c}$ is maximal, indicates the presence of superfluidity, not so much at $T_{c}$ but once superfluid order is well established at $T=0.5 T_{c}$, through the presence of sharper coherence features, much as seen in the cuprates.

These plots have important implications for interpreting predicted signatures of superfluidity, such as those [23] based on laser probing of "atomic Cooper pairs," where it has been argued that there is a conceptual analogy between normal metal-superconductor tunneling [which measures $N(\omega)$ ], and resonant scattering of laser light. The present work introduces a caution in interpreting future atomic trap experiments: Because of the presence of a pseudogap, the signatures of superfluid onset are not as simple as in BCS or the related Bogoliubov-de Gennes theory. In general one has to distinguish between the superfluid order parameter and the fermionic excitation gap. Nevertheless, superfluid coherence appears to be visible as fine structure effects in the fermionic density of states. While the fermionic contributions [via $N(\omega), n_{k}$ ] do not provide a clear indication of superfluidity, they do establish the nature of the pairing regime: BCS, BEC or PG, a "pseudogapped superfluid."

In addition to investigating and revisiting experimental signatures, the main theoretical contributions of this paper are to establish the presence and role of preformed fermion pairs at and above $T_{c}$, which evolve into noncondensed pairs below $T_{c}$. These pairs hybridize strongly with their molecular boson counterparts associated with the Feshbach resonance. In this context we present a generalized mean field treatment of the broken symmetry phase for ultracold fermionic atoms, which unlike other $T \neq 0$ approaches, connects smoothly to the conventional [9] crossover ground state. Because the Feshbach resonance has no natural analog in high $T_{c}$ systems, it remains to be seen whether the differences in these two systems will outweigh the similarities.

We acknowledge useful discussions with D. Jin and R. Hulet. This work was supported by NSF-MRSEC Grant No. DMR-0213745 (J.S. and K.L.), NSF Grants No. DMR0094981 and No. JHU-TIPAC (Q.C.), by the U.S. Department of Energy (J.N.M.), by SNS Grant PRIN 2002, Pisa, Italy (M.L.C.), and by the National Science Foundation (M.L.C. and, M.J.H.).
[1] M. Holland, S. J. J. M. F. Kokkelmans, M. L. Chiofalo, and R. Walser, Phys. Rev. Lett. 87, 120406 (2001).

[2] E. Timmermans, K. Furuya, P. W. Milonni, and A. K. Kerman, Phys. Lett. A 285, 228 (2001).

[3] M. Greiner, C. Regal, and D. Jin, Nature (London) 426, 537 (2003).

[4] S. Jochim et al., Science 302, 2101 (2003).

[5] M. Zwierlein et al., Phys. Rev. Lett. 91, 250401 (2003).

[6] J. N. Milstein, S. J. J. M. F. Kokkelmans, and M. J. Holland, Phys. Rev. A 66, 043604 (2002).

[7] Y. Ohashi and A. Griffin, Phys. Rev. Lett. 89, 130402 (2002).

[8] P. Nozières and S. Schmitt-Rink, J. Low Temp. Phys. 59, 195 (1985).

[9] A. J. Leggett, in Modern Trends in the Theory of Condensed Matter (Springer-Verlag, Berlin, 1980), pp. 13-27.

[10] J. W. Serene, Phys. Rev. B 40, 10873 (1989).

[11] J. Sofo and C. Balseiro, Phys. Rev. B 45, 8197 (1992).

[12] Q. J. Chen, I. Kosztin, B. Janko, and K. Levin, Phys. Rev. Lett.
81, 4708 (1998).

[13] R. Micnas and S. Robaszkiewicz, Condens. Matter Phys. 1, 89 (1998).

[14] T. Kostyrko and J. Ranninger, Phys. Rev. B 54, 13105 (1996).

[15] J. Stajic, A. Iyengar, Q. Chen, and K. Levin, Phys. Rev. B 68, 174517 (2003).

[16] S. Kokkelmans et al., Phys. Rev. A 65, 053617 (2002).

[17] J. Maly, B. Jankó, and K. Levin, Physica C 321, 113 (1999).

[18] A. Griffin and Y. Ohashi, Phys. Rev. A 67, 063612 (2003).

[19] Q. J. Chen, I. Kosztin, and K. Levin, Phys. Rev. Lett. 85, 2801 (2000).

[20] L. Viverit, S. Giorgini, L. Pitaevskii, and S. Stringari, e-print cond-mat/0307538.

[21] W. P. Zhang, C. A. Sackett, and R. G. Hulet, Phys. Rev. A 60, 504 (1999).

[22] M. L. Chiofalo, S. Kokkelmans, J. Milstein, and M. Holland, Phys. Rev. Lett. 88, 090402 (2002).

[23] P. Torma and P. Zoller, Phys. Rev. Lett. 85, 487 (2000). 